Sharif University of Technology
Scientia Iranica
SCIENTIA
I RAN ICA

\title{
Analysis of the secondary vortices in rotating flow induced by a twisted tape inside a pipe
}

\author{
R. Cazan and C.K. Aidun* \\ G. W. Woodruff School of Mechanical Engineering, Georgia Institute of Technology, Atlanta, Georgia, 30332, USA.
}

Received 11 February 2018; accepted 5 May 2018

\author{
KEYWORDS \\ Swirling flow; \\ Co-rotating vortex; \\ Flow through twisted \\ tape; \\ Spiral vortex in a \\ pipe.
}

\begin{abstract}
$\overline{\text { Abstract. Flow through a twisted tape (swirler) creates a complicated vortex structure }}$ downstream in the pipe. Detailed velocity measurements with Laser Doppler Velocimetry (LDV) along horizontal and vertical axes perpendicular to the axial flow direction have shown a strange flow pattern at the center of the rotating flow-a counter-rotating vortex seems to be present at the center with periodically varying magnitude in the axial direction. In highly detailed measurements, it is shown that this behavior results from a pair of co-rotating secondary vortices that are superimposed on the primary rotating flow in a helical formation. The source of these secondary vortices has remained unclear. This study presents numerical simulations of the flow through $180^{\circ}$ twisted tape in a pipe, complementing the previous experimental results. The simulations reproduce the characteristics of the helical co-rotating vortices observed in experiments and provide details of the flow field. The results provide an insight into the formation of the secondary vortices inside the twisted tape, thus explaining the experimental observations. The mechanism of the vortex formation is described, showing that the secondary co-rotating helical vortices are produced by a pair of single co-rotating vortex formed on each side of the twisted tape.
\end{abstract}

(C) 2018 Sharif University of Technology. All rights reserved.

\section{Introduction}

Swirling flows are found in many industrial applications, such as enhanced mixing of fluids, increasing heat transfer in heat exchangers, homogenizing mixtures in casting and in the chemical industry, or breaking the fuel droplets, and stabilizing flames in combustion. There are numerous swirl generation systems, most common of which are vanes, eccentric fluid injection, rotating pipes, or twisted-tape inserts.

In this study, we focus on the twisted-tape insert with the main characteristics presented in Figure 1. The defining parameters include $180^{\circ}$ pitch, $H$, pipe

\footnotetext{
*. Corresponding author.

E-mail address: cyrus.aidun@me.gatech.edu (C.K. Aidun)
}

doi: $10.24200 /$ sci. 2018.50440 .1694

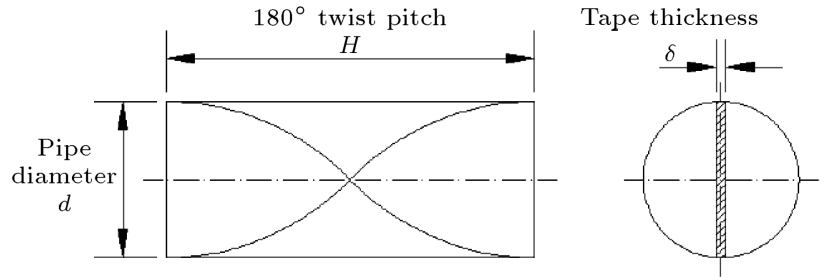

Figure 1. Twisted-tape parameters.

diameter, $d$, and tape thickness, $\delta$. The relevant nondimensional parameters include twist ratio, $y_{r}=H / d$, and Reynolds number, $\operatorname{Re}=d \cdot U_{b} / v$, where $U_{b}$ is the bulk velocity (based on flow rate and cross-sectional area), and $v$ is the kinematic viscosity of water. Low values of ratio $y_{r}$ correspond to strong twist and high swirl numbers.

There are numerous studies dedicated to the twisted tape; however, most studies investigate only the 
variations of the heat transfer and friction coefficient with few attempts to outline a detailed flow structure. A comprehensive list of papers regarding twisted tape inserts is summarized in the review article of Dewan et al. [1].

Early investigations of the flow field in pipes with full-length twisted-tape inserts were done with intrusive probes inserted directly into the flow [2-4], limiting the accuracy of the results. Seymour's measurements [3] revealed the existence of a secondary flow with a double vortex structure at Reynolds number as high as $3 \times 10^{5}$.

Date [5] analyzed the flow in the laminar regime using a vorticity-stream function formulation. He used a rotating coordinate system and assumed fully developed flow with no axial gradients. The contours of the axial velocity profile displayed one peak for two tapes with a lower twist ratio and two peaks for a tape with a higher twist ratio. No tangential velocity results were shown.

Other investigators [6-9] used both numerical simulations and smoke flow visualizations to study the secondary motion in swirling air flow. Their images show the presence of two structures in the semicircular cross-section, which they identified as counterrotating vortices. The experimental photos from these studies clearly show the spiral of a co-rotating vortex at the leading corner; however, the rest of the flow does not show a clear counter-rotation, as claimed. The smoke visualizations at low Reynolds number are compared to numerical simulations using a vorticitystream function formulation. Their simulations show two counter-rotating vortices similar to those presented by Seymour [3].

Kazuhisa et al. [10] described another numerical investigation of the laminar swirling flow generated by a twisted-tape insert in a cylindrical pipe. Their model employs a non-orthogonal coordinate system rotating with the tape, while the equations are solved using the
SIMPLEC algorithm [11]. Their velocity vector plots show either one vortex rotating counter to the twist of the tape or two counter-rotating vortices.

Through detailed Laser Doppler Velocimetry (LDV) measurements, Cazan and Aidun [12] showed that the unexpected counter-rotating flow found in the LDV measurements near the pipe centerline in not the continuation of counter-rotating vortices formed inside the insert, as elucidated in previous studies [3,6,9]. In fact, the LDV measurements of Cazan and Aidun [12] did not indicate the presence of a counter-rotating vortex immediately downstream at the trailing edge of the twisted tape. The LDV measurements and air bubble visualizations [12] show two stable co-rotating helical vortices downstream of $180^{\circ}$ twisted tapes for $\operatorname{Re}>10^{4}$ (Figure 2). The helical secondary vortices are rotating in the same direction with the main flow; in addition, the helix winding is in the same direction-the co-rotating vortices, the helix formed by the vortices, and the main flow all have positive or clockwise rotation, as shown in Figure 2. The pitch of the helix formed by the co-rotating vortices is found to be independent of Re. Near the pipe centerline, the two co-rotating helical vortices create an apparent counter-rotating non-axisymmetric flow region. It is, therefore, the interaction of the two corotating vortices that induces the apparent counterrotating flow at the pipe centerline observed in the LDV measurements [12]. This is confirmed by highspeed visualizations of air bubbles injected upstream, showing the flow field induced by the interaction of two co-rotating helical vortices. The stationary and stable character of the co-rotating vortices (also observed in previous studies $[13,14]$ ) allowed a detailed investigation of the flow field.

The details of the secondary flow generated inside the twisted tape have remained unclear. Experimental investigations inside the twisted tape are difficult and unreliable as probes inserted into the swirling flow alter

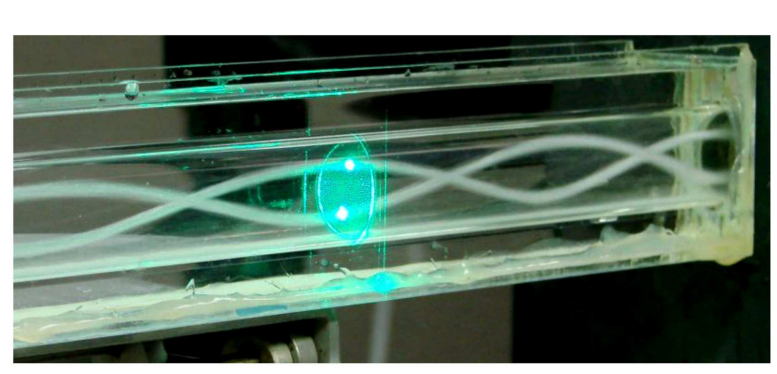

(a)

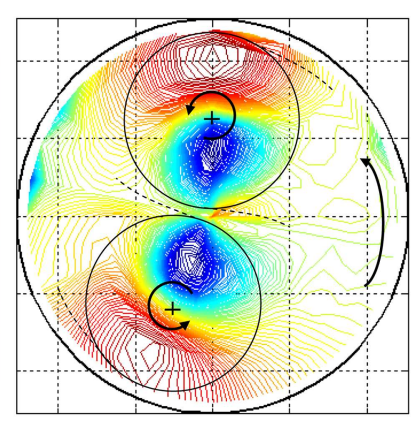

(b)

Figure 2. Co-rotating helical vortices induced by a $180^{\circ}$ twisted-tape with a twist ratio of $y_{r}=2.36(60 \mathrm{~mm}$ pitch $)$ at $\operatorname{Re}=7.7 \times 10^{4}$ (bulk velocity of $U_{b}=3 \mathrm{~m} / \mathrm{s}$ ) in a pipe with a diameter of $d=25.4 \mathrm{~mm}$ : (a) Air bubble visualization (the flow is from right to left), (b) average tangential velocity contours of the co-rotating secondary vortices after subtracting the main vortex background. The circles show the approximate boundary of the vortices, while the arrows show their rotation (from LDV measurements [12]). 
the flow. LDV measurements around the twisted tape are also challenging. The present article describes a numerical study complementing the previous experiments [12], focusing on the formation of the secondary helical vortices.

\section{Numerical model}

The majority of the numerical studies investigate the flow inside the twisted tape at low Re, while the helical vortices are observed experimentally downstream of the twisted tape at Re greater than $10^{4}$. This explains the absence of any previous reports of helical vortices induced by twisted tapes. In the present study, the secondary flow is investigated by simulating the swirling flow through a $180^{\circ}$ twisted tape with a simple straight pipe section upstream of the twisted tape and another straight pipe section downstream of the twisted tape. The flow is calculated using the commercial CFD software FLUENT (ANSYS Fluent, ANSYS Inc.). There are previous reports of successful numerical simulations using FLUENT for flows through static mixers, which consist of a series of left and right helical elements placed at right angles to each other $[15,16]$. Each helical element in a static mixer is basically a $180^{\circ}$ twisted tape; however, the alternating helical elements destroy the secondary structures.

\subsection{Governing equations}

The stability of the helical vortices observed during the experiments (visible in the movies accompanying the on-line version of Cazan and Aidun [12]) suggests that the mean (Reynolds averaged) flow is in a quasi-steady state. The working fluid in the model is liquid water (incompressible) at room temperature. The flow field is obtained solving the governing integral equations for the conservation of mass and momentum using a pressure-based solver, in which the pressure field is extracted by solving a pressure correction equation.

For a steady incompressible flow, the mass conservation equation in Cartesian coordinates expressed in index notation (where repeated indices imply summation) is:

$$
\frac{\partial u_{i}}{\partial x_{i}}=0
$$

where $u_{i}$ represents the velocity component in direction $x_{i}$. The momentum conservation equation is given by:

$$
\rho \frac{\partial\left(u_{i} u_{j}\right)}{\partial x_{j}}=-\frac{\partial p}{\partial x_{i}}+\frac{\partial \tau_{i j}}{\partial x_{j}}+\rho g_{i},
$$

where $\rho$ is the density, $p$ is the pressure, and $g$ is gravity. The stress tensor, $\tau_{i j}$, is given by:

$$
\tau_{i j}=\mu\left(\frac{\partial u_{i}}{\partial x_{j}}+\frac{\partial u_{j}}{\partial x_{i}}\right),
$$

where $\mu$ is the dynamic viscosity.

In the experimental data reported by Cazan and Aidun [12], a honeycomb and a 9:1 contraction are located immediately upstream of the twisted tape to reduce turbulent fluctuations. Both Direct Numerical Simulations (DNS), based on Eqs. (1)-(3), and simulations based on the Reynolds Averaged Navier-Stokes (RANS) equation are presented which are given by

$$
\begin{aligned}
\rho \frac{\partial\left(u_{i} u_{j}\right)}{\partial x_{j}}= & \frac{\partial}{\partial x_{j}}\left[\mu\left(\frac{\partial u_{i}}{\partial x_{j}}+\frac{\partial u_{j}}{\partial x_{i}}\right)\right]-\frac{\partial p}{\partial x_{i}}+\rho g_{i} \\
& +\frac{\partial}{\partial x_{j}}\left(-\rho \overline{u_{i}^{\prime} u_{j}^{\prime}}\right),
\end{aligned}
$$

where $\rho \overline{u_{i}^{\prime} u_{j}^{\prime}}$ is the Reynolds stress based on crosscorrelation of the velocity fluctuations, $u_{i}^{\prime}$. The large gradients in the flow and the 3D nature associated with the helical vortices require a fine grid with a large number of computational cells on the Kolmogorov length scale. DNS on the required scale to resolve the dissipative scale is prohibitively expensive. However, we use coarser scale DNS of flow in addition to solving the RANS equation, where the Reynolds stresses $\rho \overline{u_{i}^{\prime} u_{j}^{\prime}}$ are modeled. For swirling flows where turbulence is anisotropic, the "Reynolds Stress Model" (RSM), which solves the transport equation for each component of the Reynolds stress tensor, provides reasonable results (for details of RSM, see Pope [17]).

The flow length scale is the pipe diameter, $d=$ $25.4 \mathrm{~mm}$; the velocities are normalized with the bulk velocity, $U_{b}=\frac{v \cdot \operatorname{Re}}{d}$; and the kinematic viscosity is set to $v=10^{-6} \mathrm{~m}^{2} / \mathrm{s}$ for water.

\subsection{Computational domain}

The flow is three-dimensional and non-axisymmetric. The numerical model simulates a cylindrical pipe of 1 inch $(25.4 \mathrm{~mm})$ diameter with a twisted-tape insert using the finite volume method. The computational domain employs a 3D Cartesian coordinate system $X Y Z$ centered on the pipe axis at the downstream edge of the twisted tape with $Z$-axis orientated along the pipe axial direction (Figure 3 ).

The mesh is an unstructured tetrahedral cell selected for their capacity to accommodate the complex 3D shape of the twisted tape. Grid independence tests for grids with $0.039,0.027$, and 0.021 average side-length cells indicate that 0.027 cells represent the optimum for these simulations. Grids with 0.039 cells underestimate the pitch of the secondary vortices, while those with 0.027 and 0.021 provide similar results.

The mesh with 0.027 cells contains approximately 4 million tetrahedral cells. The $17.72 d$ long computational domain (equivalent to $450 \mathrm{~mm}$ for the pipe with $d=25.4 \mathrm{~mm}$ ) has three sections: a $1.57 d$ long straight pipe inlet section followed by a pipe section containing the $180^{\circ}$ twisted-tape insert with twist ratios $1.77,2.36$ 


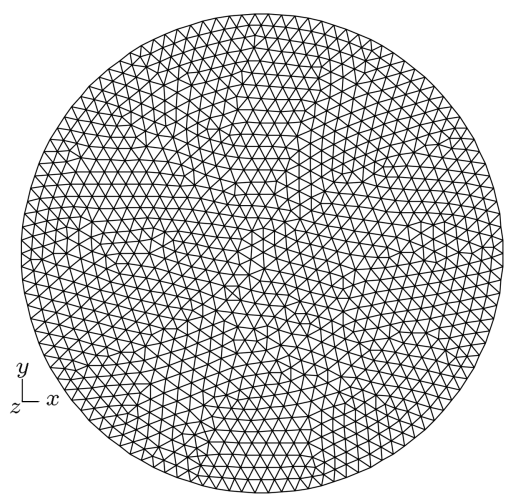

(a)
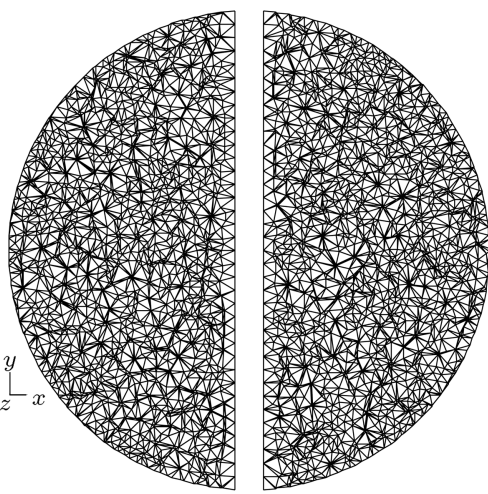

(b)

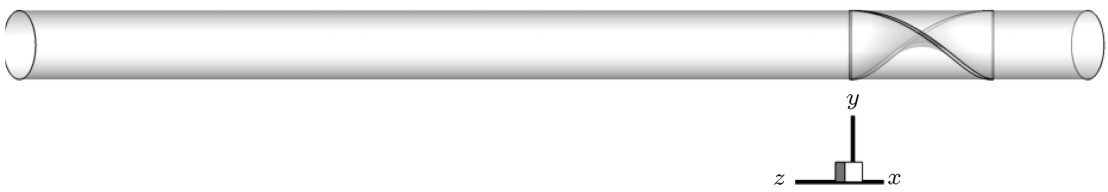

(c)

Figure 3. Computational domain for the twisted tape: (a) Inlet cross-section, (b) cross-section showing the inlet of the twisted-tape, and (c) side view of the $17.72 d$ long computational domain.

or $3.54(45,60$ or $90 \mathrm{~mm}$ long) and ending with a straight pipe section corresponding to the experimental test section. The thickness of the twisted tape is uniform and equal to $0.059 \mathrm{~d}(1.5 \mathrm{~mm})$. Figure 3 shows the grid cross-section at the pipe inlet, the cross-section at the twisted-tape inlet, and a side view of the entire domain.

\subsection{Numerical solver}

The equations are solved using the commercial CFD software FLUENT. The solver is 3D, pressure-based, segregated, and implicit. The gradients are evaluated using the node-based Green-Gauss method, which provides second-order spatial accuracy and improved results for unstructured tetrahedral meshes compared with the cell-based scheme $[18,19]$. The momentum equation is discretized using a MUSCLE scheme [20] (Monotone Upstream-Centered Schemes for Conservation Laws). The pressure velocity coupling is implemented using the PISO (Pressure-Implicit with Splitting of Operators) method with skewness correction, which is part of the SIMPLE family of algorithms [11]. The PISO coupling scheme provides faster convergence on meshes with a high degree of distortion [21]. The pressure discretization is implemented using the PRESTO method (PREssure STaggering Option), which provides improved accuracy for flows with high swirl numbers, high-speed rotating flows, and flows in strongly curved domains compared to the other models available [22].

The flow is solved with both coarse-mesh DNS and RANS modeling simulations. With the RANS equations, the Reynolds stress is modeled using the well-known Reynolds Stress Model (RSM) [23], which is recommended for swirling flows and flows with secondary motion where the turbulence is anisotropic [17].

At the walls, the near-wall Reynolds stresses and the dissipation rate are calculated with the standard wall functions, as proposed by Launder and Spalding [24]. The stresses are specified explicitly assuming that equilibrium and the log-law are valid near the walls, while convection and diffusion are neglected in the stress transport equations.

No-slip boundary conditions are applied on solid surfaces (on the wall and twisted tape). The inflow velocity profile was determined experimentally by running a test with a simple pipe, without the twisted tape. The experiments showed that, despite the large Reynolds number used, the honeycomb flow straightener and the 9:1 contraction maintained the flow at close to low turbulent fluctuation at the twistedtape inlet for the entire velocity range investigated. The experimental inlet profile used in the simulations is flat with specified axial inlet velocities $V_{z} / U_{b}=1$ (Figure 4) for all Re investigated. The turbulent intensity at the inlet determined from experiments is about $1 \%$ for the turbulent simulations [12].

The solution is considered satisfactory when convergence criterion $\varepsilon_{i}<10^{-3}$ is satisfied, where $\varepsilon_{i}$ represents the relative errors for the three momentum equations along each coordinate axis and the mass conservation. For RANS simulations, the residuals of the six components of the Reynolds stress tensor are monitored in addition to the residuals of mass 


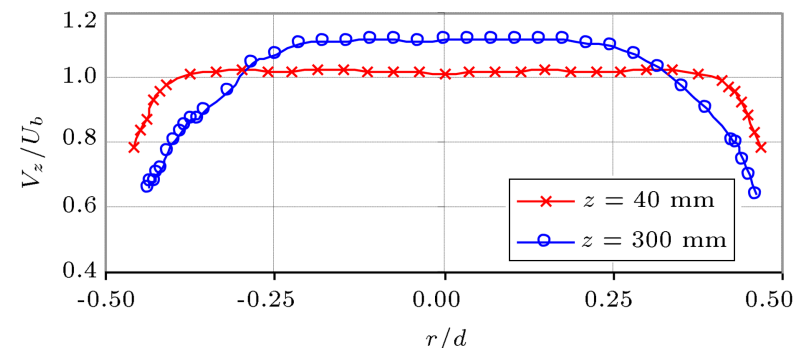

(a)

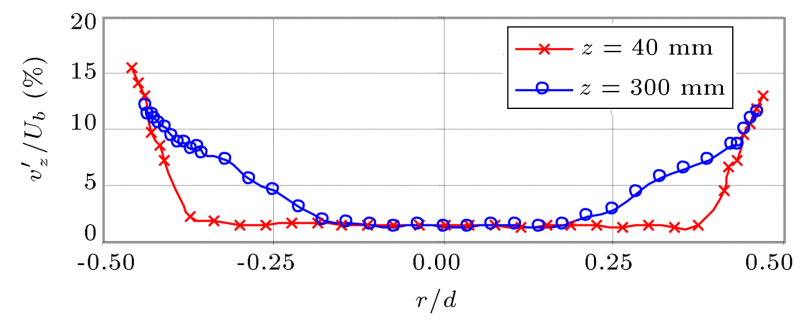

(b)

Figure 4. Normalized average axial velocity $V_{z} / U_{b}$ (a) and normalized rms fluctuations of the axial velocity $v_{z}^{\prime} / U_{b}(\mathrm{~b})$ at $\operatorname{Re}=7.7 \times 10^{4}\left(U_{b}=3 \mathrm{~m} / \mathrm{s}\right)$ in the absence of the twisted tape at two axial locations, $z=40 \mathrm{~mm}$ $(1.57 d)$ and $z=300 \mathrm{~mm}(11.81 d)$ downstream of the contraction end (from LDV measurements [12]).

and momentum. Comparisons with experimental data are also used to assess the accuracy of the numerical results.

\section{Results and comments}

\subsection{Model validation}

The numerical model is validated by comparing the pitch of the helical vortices (qualitative) and the tangential velocity profile (quantitative) with flow visualization and LDV measurements, respectively. Figure 5 shows numerical results obtained through the coarsemesh DNS simulation for the twisted tape with twist ratio $y_{r}=2.36(60 \mathrm{~mm}$ pitch tape $)$ at $\operatorname{Re}=7.7 \times 10^{4}$ (bulk velocity $3 \mathrm{~m} / \mathrm{s}$ ) side by side with a photo recorded during experimental tests. The helical vortices are identified from the numerical simulation results by isobar surfaces of low-pressure region, corresponding to the location of the air bubble accumulation observed in the experiments.

By comparing the pitch of the helical vortices and their axial and radial positions inside the pipe, the results of the coarse-mesh DNS match the experimental images both in the developing region inside the twisted tape and in the stable region inside the straight pipe. Inside the straight pipe, the pitch of the helical vortices is approximately $3.15 d(80 \mathrm{~mm})$ both in the experiments and in the numerical simulations.

The reference value of $p=1$ for the isobar surfaces used for this case to identify the helical vortices is selected to match approximately the thickness of the bubble streams observed in experiments. The pathlines calculated numerically (Figure 5(b)) also match well with the few short pathlines of small air bubbles visible in the experimental visualization, as a result of the fast flow motion relative to the exposure time of 4 milliseconds (Figure 5(a)).

Similar comparisons showing the entire experimental test section for the flows induced by all the twisted tapes available $\left(y_{r}=1.77,2.36\right.$ and 3.54$)$ at $\operatorname{Re}=7.7 \times 10^{4}$ are shown in Figures 6 to 8 . The pitch and position of the helical vortices predicted by the coarse-mesh DNS are in good agreement with the experiments.

The pitch of the helical vortices is approximately $2.36(60 \mathrm{~mm})$ for the twisted tape with $y_{r}=1.77,3.15$ $(80 \mathrm{~mm})$ for the twisted tape with $y_{r}=2.36$ and 4.72

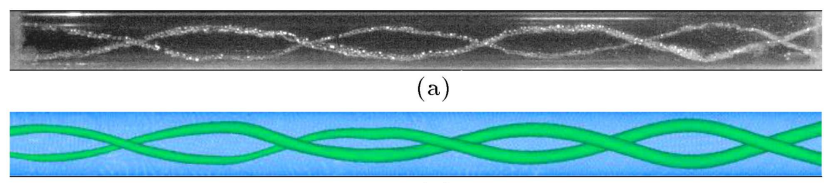

(b)

Figure 6. Helical vortices generated by a twisted tape with twist ratio of $y_{r}=1.77$ ( $45 \mathrm{~mm}$ pitch) at $\operatorname{Re}=7.7 \times 10^{4}$ from (a) high speed visualization and (b) numerical results.

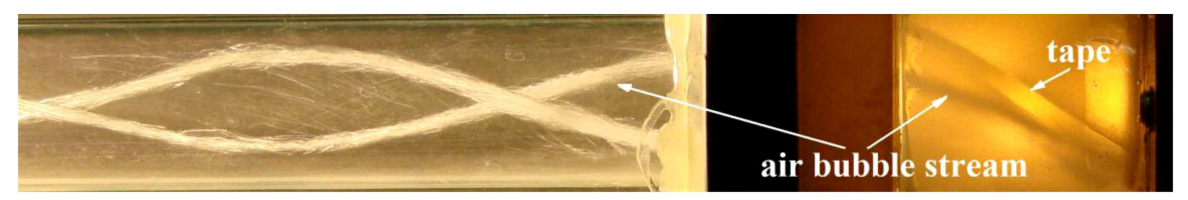

(a)

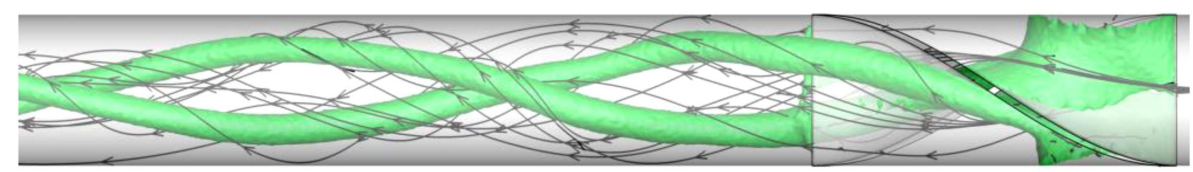

(b)

Figure 5. Experimental and numerical visualizations of the development of the helical vortices for the twisted tape with $y_{r}=2.36$ at $\operatorname{Re}=7.7 \times 10^{4}:$ (a) Side-view photo of air bubble streams, and (b) side view plot of isobar surfaces $(p=1)$ and pathlines calculated from DNS results. 


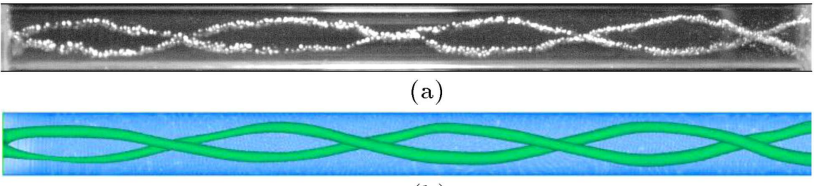

(b)

Figure 7. Helical vortices generated by a twisted tape with a twist ratio of $y_{r}=2.36(60 \mathrm{~mm}$ pitch $)$ at $\operatorname{Re}=7.7 \times 10^{4}$ with $(\mathrm{a})$ high speed visualization, and (b) numerical results.

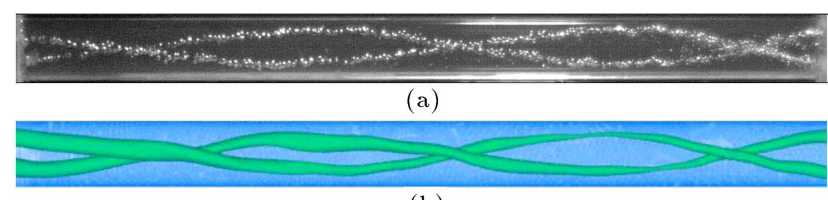

(b)

Figure 8. Helical vortices generated by a twisted-tape with a twist ratio of $y_{r}=3.54$ (90 $\mathrm{mm}$ pitch) at $\mathrm{Re}=7.7 \times 10^{4}$ with (a) high speed visualization and (b) numerical results.

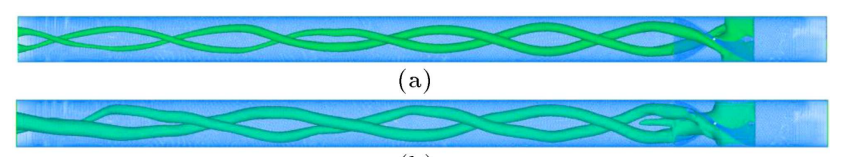

Figure 9. Helical vortices induced by a twisted tape with $y_{r}=1.77$ at $\operatorname{Re}=7.7 \times 10^{4}$ : (a) Isobar surfaces of $p=1$ for the DNS simulations, and (b) isobar surfaces of $p=1$ for the RANS simulation.

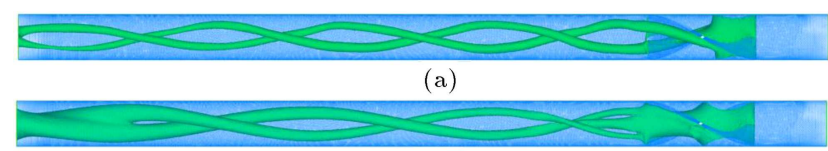

(b)

Figure 10. Helical vortices induced by a twisted-tape with $y_{r}=2.36$ at $\operatorname{Re}=7.7 \times 10^{4}$ : (a) Isobar surfaces of $p=1$ for the DNS simulation, and (b) isobar surfaces of $p=1$ for the RANS simulation.

(120 mm) for the twisted tape with $y_{r}=3.54$, both in the experiments and in the numerical simulations. The reference values for the isobar surfaces, which identify the vortex cores, are at $p=1$ for all twisted tapes.

Figures 9 and 10 allow a comparison between the pitch of the helical vortices induced by the twisted tape with $y_{r}=1.77$ and $y_{r}=2.36$ calculated with both DNS and RANS simulations. As shown before, the DNS predicts the characteristic pitch of the co-rotating vortices observed in the experiments. The RANS simulation, however, shows significant discrepancies with the experiments.

The pitch determined using the turbulent simulation is approximately $25 \%$ longer for both the twisted tape with $y_{r}=1.77$ (75 mm pitch instead of $60 \mathrm{~mm}$ measured in experiments) and the twisted tape with $y_{r}=2.36(100 \mathrm{~mm}$ instead of $80 \mathrm{~mm}$ measured in experiments). Once the vortices are out of the twisted tape, the pitch seems to be preserved to a relatively constant value inside the straight pipe for both the laminar and turbulent models. As such, the error in the turbulent model is likely due to a slower vortex development inside the twisted tape in the turbulent model, not due to excessive dissipation inside the straight pipe. The flow entering the twisted tape is laminar, while the flow inside the straight pipe is turbulent; thus, the vortices are generated in a transitional flow inside the twisted tape.

The fact that the pitch does not vary with $R e$ observed in the experiments suggests that turbulence does not have a significant effect on the helical vortices for the $14 d$ test section starting from the downstream end of the twisted tape. These results show that the DNS provides a more accurate description of the transitional flow involved in the vortex generation, or in other words, the RANS simulations overpredict the effect of turbulent fluctuations.

In addition to the qualitative comparison with the experimental visualization, the numerical models are also validated quantitatively against the LDV measurements. Figure 11 shows a comparison between the experimental measurements of the average tangential velocity profiles and tangential velocity profiles calculated with DNS across the horizontal diameter at two axial locations. The comparison between experimental

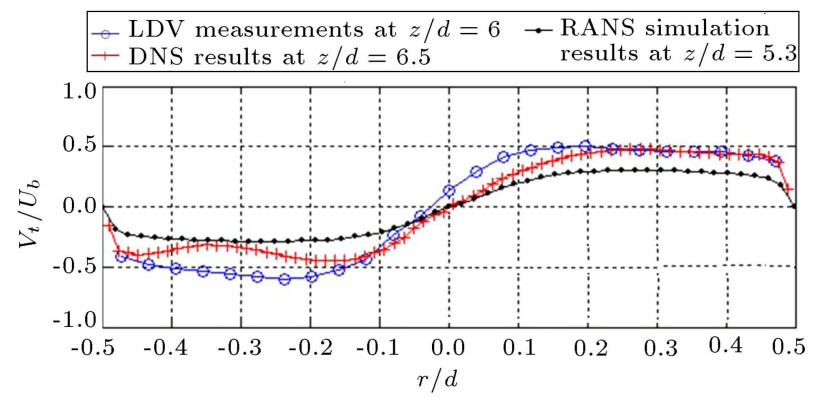

(a)

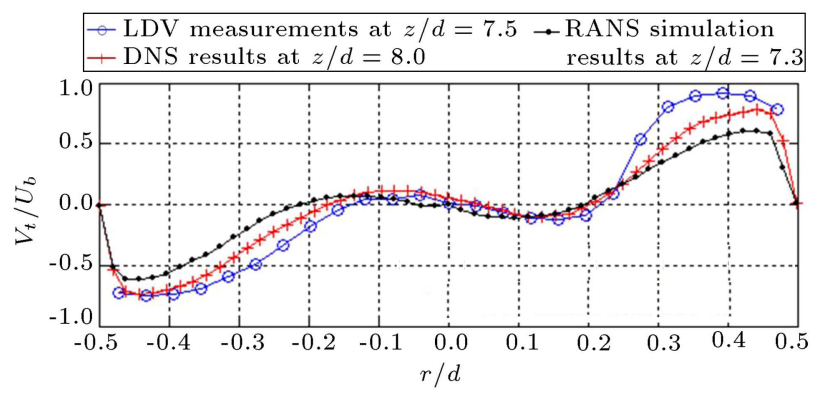

(b)

Figure 11. Comparison between experimental average tangential velocity profiles and calculated tangential velocity profiles from the numerical simulation for the tape with $y_{r}=2.36$ at $\operatorname{Re}=7.7 \times 10^{4}\left(U_{b}=3 \mathrm{~m} / \mathrm{s}\right):$ (a) Between the helical co-rotating vortices and (b) through the helical co-rotating vortices. 
and numerical results is shown at two axial locationsone location where the co-rotating helical vortices are in a vertical position (i.e., on top of each other), and the second location where the co-rotating helical vortices are in a horizontal position (i.e., side-by-side of each other). When the helical vortices are in a vertical position (or on top of each other), the velocities are measured between the helical vortices (Figure 2), since the line of LDV measurement is along the $x$ axis (horizontal). When the helical vortices are in a horizontal position, the velocities are measured through the helical vortices (Figure 2). Since the pitch is not exactly the same in all cases, the axial location for each case could vary as much as $10 \%$.

The tangential velocity profiles from DNS results match the trends and the maximum values observed experimentally, yet with a small $0.6 d$ axial displacement, while the average tangential velocity profiles calculated with the RANS model capture the trends from the experimental measurements and, yet, show more diffused and weaker co-rotating vortices. The underestimate of the vortex strength and the overprediction of the pitch result from the underestimation of the vortex strength inside the twisted tape by the RANS model. The characteristic of the helical vortices downstream of the twisted tape is sensitive to the early development of the vortices inside the twisted tape.

The variation of the normalized tangential velocity with $\operatorname{Re}$ for the entire range of Re from $10^{4}$ to $10^{5}$ by the experimental measurements and DNS is presented in Figure 12. The tangential velocities are normalized with the bulk velocity corresponding to Re. The profiles collected through the co-rotating helical vortices display the counter-rotating flow at the center. The numerical results at $z / d=8.03$ (Figure 12(b)) match well with the experimental profiles measured at $z / d=7.48$ (Figure 12(a)). The DNS captures the change in the velocity profile at $\operatorname{Re}=10^{4}$, but not its magnitude.

\subsection{Vortex identification}

Previously, the helical vortices have been identified in the numerical simulation results by isobar surfaces of low pressure [25] which correspond to the region of air bubble accumulation in the experimental flow visualizations. That method is confirmed in this section by the closed streamline [25,26] and $\lambda_{2}$ method [27].

Figure 13 shows the velocity vectors and the streamlines in the cross-section plane at $z / d=6.57$ for the flow induced by a twisted tape with $y_{r}=2.36$ at $\operatorname{Re}=7.7 \times 10^{4}$. The closed streamlines indicate the presence of the helical vortices and, also, the main rotation around the pipe centerline, all in clockwise (positive) rotation. The primary vortex (or main swirl) is not identified by the low-pressure method, as the

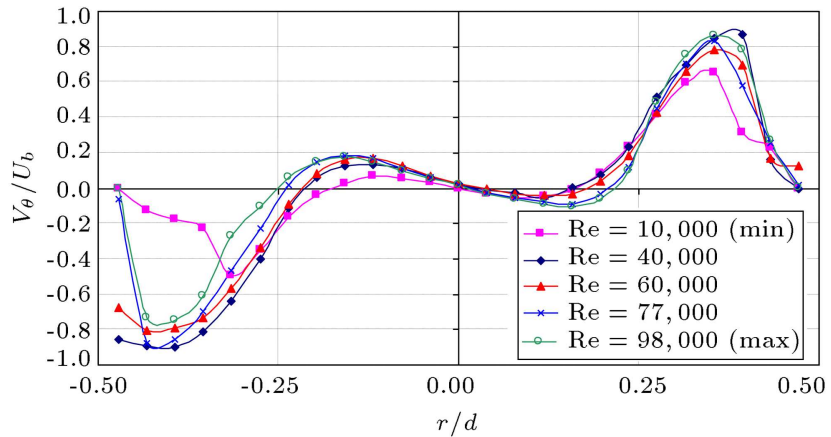

(a)

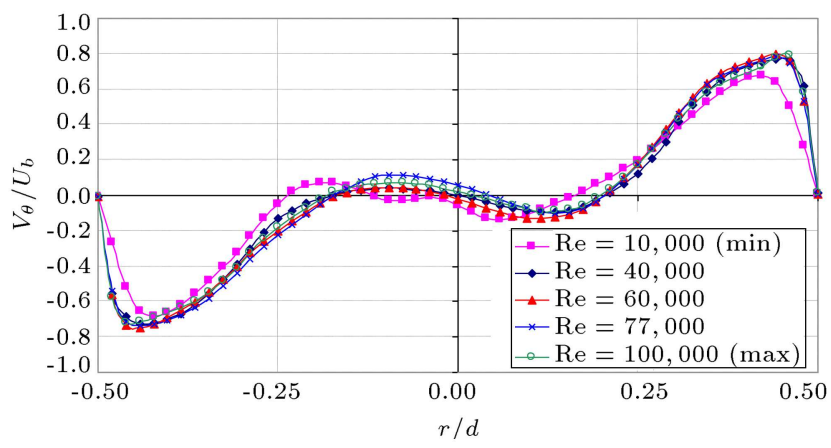

(b)

Figure 12. Variation of the normalized tangential velocity with Re: (a) Experimental profiles at $z / d=7.48$, and (b) numerical results at $z / d=8.03$. In both cases, the profiles collected through the co-rotating helical vortices display counter-rotating flow, as reported in previous studies $[12,28]$.

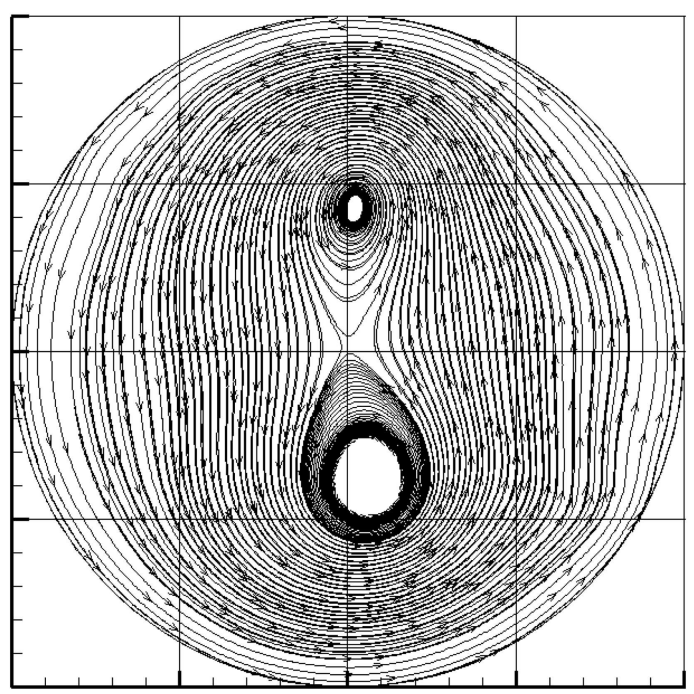

Figure 13. Stream lines visualization of the velocity field in the cross-section plane $z / d=6.57$ in the flow induced by a twisted-tape with $y_{r}=2.36$ at $\operatorname{Re}=7.7 \times 10^{4}$.

pressure is the lowest in the cores of the two co-rotating vortices.

In Figure 14, the cores of the secondary vortices are identified by $\lambda_{2}$ method in addition to the isobar surfaces of low pressure, as done in the previous 


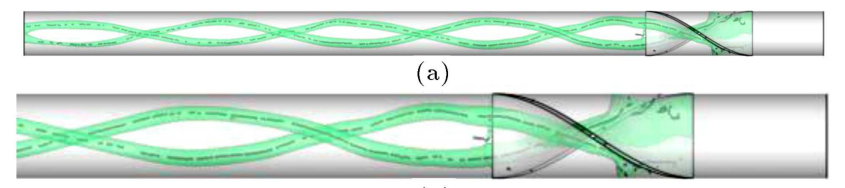

(b)

Figure 14. Comparison between vortex identification with isobar surfaces and $\lambda_{2}$ lines for (a) full domain and (b) close-up view of the twisted-tape.

section. This figure shows the entire domain, as well as a twisted-tape close-up, where the centers of the connected regions with $\lambda_{2}<0$ are marked by line segments. The isobar surfaces have been made transparent, which allows $\lambda_{2}$ lines to be visible at the centers of the low-pressure isobar surfaces.

The plots confirm that the isobar surfaces are reliable in identifying the vortex cores at high Re, where the pressure gradients generated by the swirling motion are larger than viscous effects. All the identification methods used confirm that the large, stable structures that intuitively were considered vortices based on the velocity vectors in the cross-section are actually vortices according to generally accepted vortex identification criteria. These results confirm that the air bubbles visualized in the experiments are indeed showing co-rotating helical vortices in the swirling flow induced by short twisted tapes. The isobar surface remains the most convenient method for this investigation as it allows a direct comparison between the numerical results and the experimental visualizations.

\section{Flow field analysis}

As the helical vortices behind twisted tapes have never been observed before, the numerical simulation results will be used next to provide an in-depth analysis of their behavior and their interaction with the main swirl. The advantage offered by the numerical simulation compared to the experimental investigation is that it provides entire the flow variables throughout the domain.

Figure 15 shows a perspective view of the flow field induced by a twisted-tape with $y_{r}=2.36$ inside a straight pipe at $\operatorname{Re}=7.7 \times 10^{4}$ calculated with $\mathrm{DNS}$. The cross-sectional plane shows the velocity vectors, while the pathline of particles originating along a horizontal line ( $x$-axis) at inlet of the pipe reveals the complex 3D motion created by the interaction of the two co-rotating vortices and the main swirl. The isobar surfaces of $p=1$ mark the cores of the helical vortices.

The presence of the helical vortices downstream of twisted tape was first indicated by the presence of counter-rotating flow in LDV measurements $[28,29]$. Later, the velocity field was recreated from the experimental velocity plots by Cazan and Aidun [12].

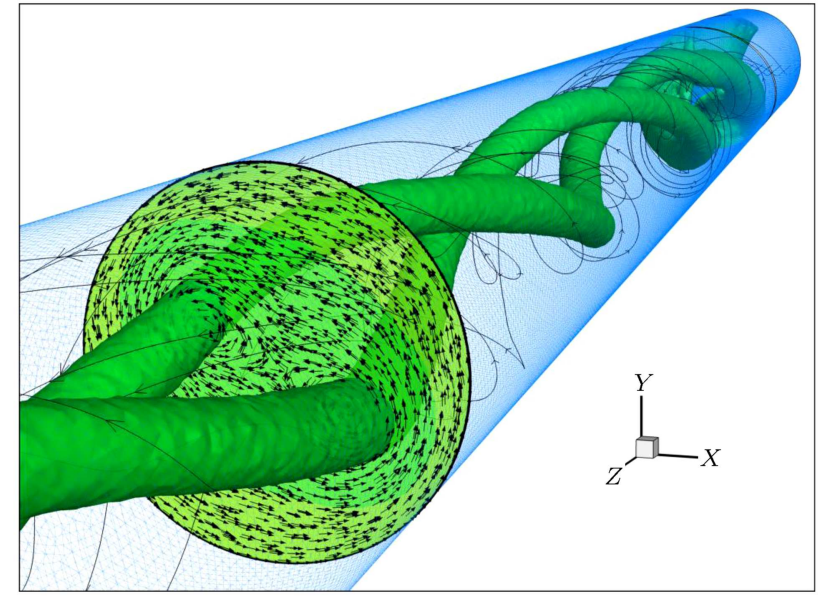

Figure 15. Isobar surfaces, pathlines, and velocity vectors identifying helical co-rotating vortices in the flow field induced by a twisted tape with $y_{r}=2.36$ at $\operatorname{Re}=7.7 \times 10^{4}$ calculated with DNS (perspective view).

Figure 16(a) shows that the velocity field reconstruction [12] is correct, as the velocity field calculated with the laminar simulation is qualitatively similar to the one reconstructed from the LDV measurements.

Figures 16(b) shows the tangential (azimuthal) velocity distribution along the horizontal $(x)$ and vertical $(y)$ axes. The velocity profile measured through the co-rotating vortices displays a distinct counter-rotating flow at the center of the pipe. The azimuthal (positive) component of velocity induced by the main swirl is small near the pipe centerline. However, the tangential velocity component induced by the co-rotating helical vortices in the opposite direction (negative) near the centerline is much stronger compared to the main swirl. The net effect is a counter-rotation to the main swirl at the central core of the pipe. The counter-rotating region is not axisymmetric, that is, the azimuthal velocity along the (horizontal) $x$-axis increases to a minimum (negative) when the co-rotating helical vortices are placed side by side along the $x$-axis, and it increases to a maximum (positive) when they are on top of each other.

Figure 16(c) shows the pressure distribution where the effect of the co-rotating vortices is clearly visible. Large pressure drop inside the cores of the helical vortices is responsible for the air bubble accumulations in the experimental visualizations. The pressure distribution shows that the two vortices have slightly different strengths, possible as an effect of the twisted-tape wake at the exit of the twisted tape. The presence of the helical vortices also reduces the axial velocity, which has maxima between the vortices and minima inside the co-rotating vortices (Figure 16(d)).

\subsection{Vortex inception and development}

The main goal of the numerical simulations presented here is to verify the hypothesis proposed in our previ- 


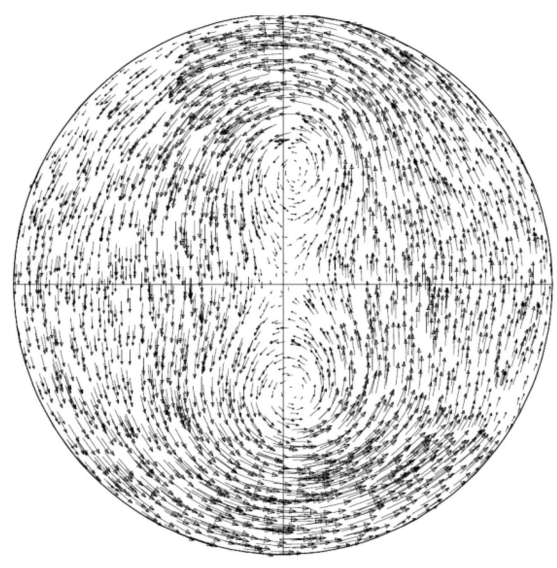

(a)

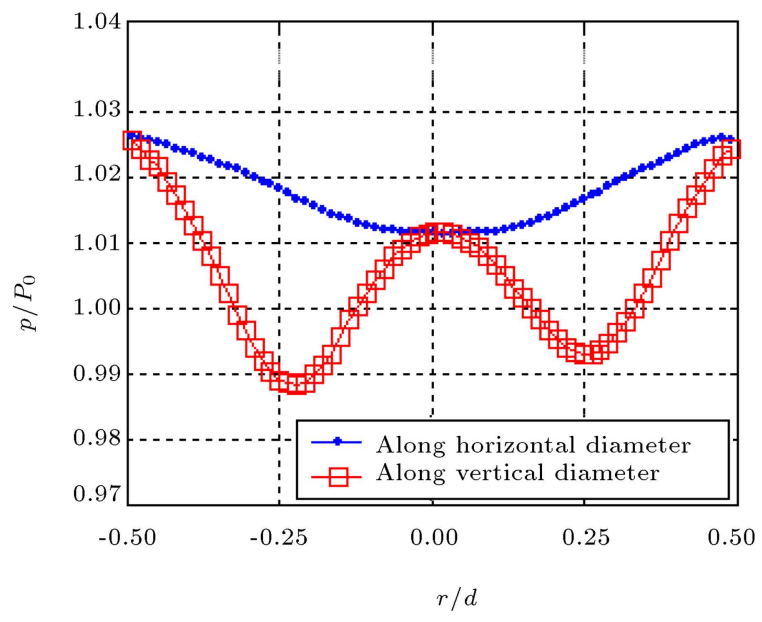

(c)

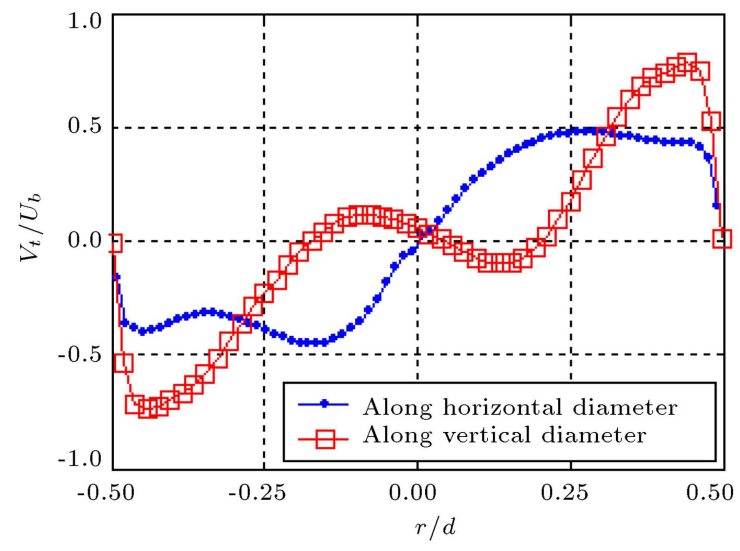

(b)

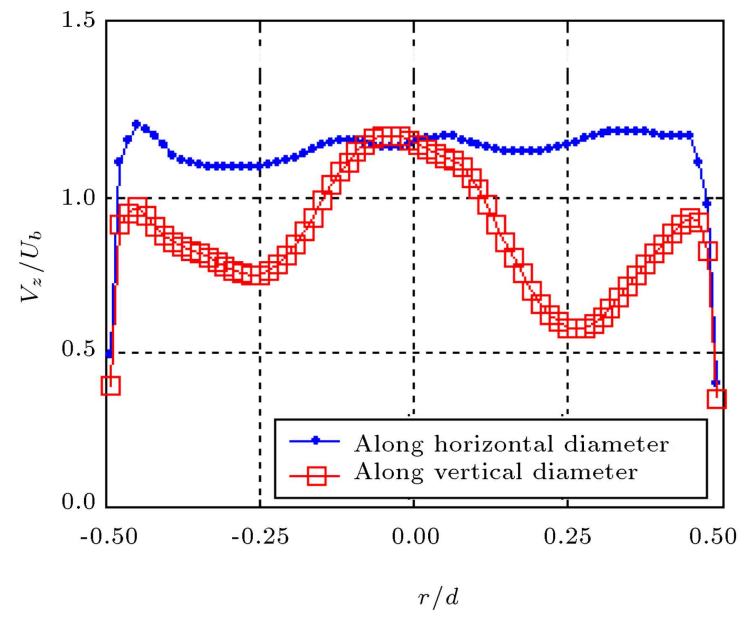

(d)

Figure 16. Variation of the flow parameters between and through the helical vortices (along the horizontal and vertical diameters, respectively) for the flow induced by a twisted tape with $y_{r}=2.36$ at $\operatorname{Re}=7.7 \times 10^{4}\left(U_{b}=3 \mathrm{~m} / \mathrm{s}\right)$ in the cross-section plane at $z / d=6.57$ downstream of the twisted-tape showing (a) cross-sectional velocity vector plot, (b) azimuthal velocity, (c) pressure, and (d) axial velocity.

ous article [12] that the helical vortices evolve from pairs of counter-rotating vortices, formed inside the twisted tape. That hypothesis attempted to match the experimental LDV measurements showing only co-rotating vortices immediately downstream of the twisted tape with results published by others presented in the Introduction. The high resolution of the velocity field calculated with the numerical model allows a detailed investigation of the flow behavior leading to the vortex formation and of the flow characteristics of the helical co-rotating vortices downstream of the twisted tape. As the flow behavior downstream of the twisted tape matches very well with the experimental observations, it is assumed here that the description of the vortex formation inside the twisted tape is equally accurate.

Velocity vector plots in cross-section planes in Figure 17 show the flow development inside the twisted tape from the inlet to downstream of the swirling inside the straight pipe. In this case, the twisted tape has a twist ratio, $y_{r}=2.36,(60 \mathrm{~mm}$ pitch $)$ at $\operatorname{Re}=7.7 \times 10^{4}$ (bulk velocity of $3 \mathrm{~m} / \mathrm{s}$ ). The plots show that the helical vortices actually evolve from single co-rotating vortices and not from pairs of counter-rotating vortices, as hypothesized before.

In order to make the figures clear, the positions of the cross-section planes are expressed both as angle of twist and as the corresponding physical distance from inlet plane.

As the fluid enters the twisted tape, the no-slip at the tape surface (Figure 17(a) and (b)) creates vorticity and pressure gradient within each section. As the tape continues to twist, the flow along the tape creates large centrifugal forces near the axis projecting the flow toward the pipe wall (Figure $17(\mathrm{e})$ and (f)). The lowpressure region at the corner with the rotation increases 


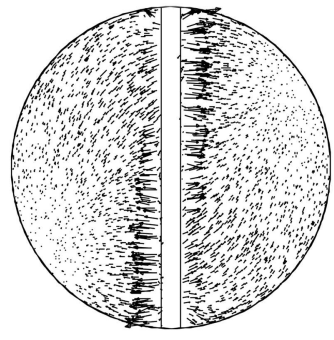

(a) Velocity vector plot at inlet plane

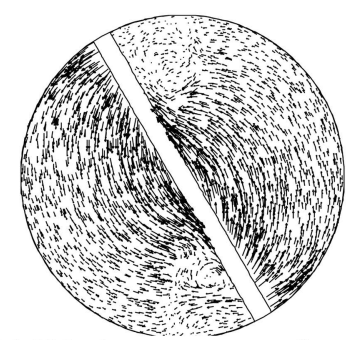

(e) Velocity vectors at $30^{\circ}$ twist (10 $\mathrm{mm}$ inside the twisted-tape)

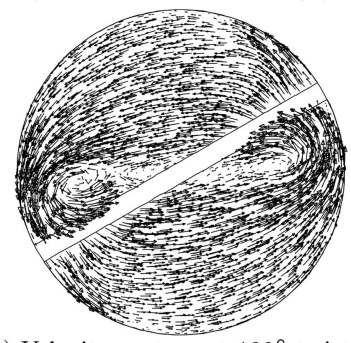

(i) Velocity vectors at $120^{\circ}$ twist (40 mm inside the twisted-tape)

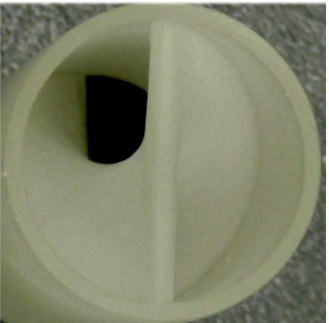

(b) Twisted-tape

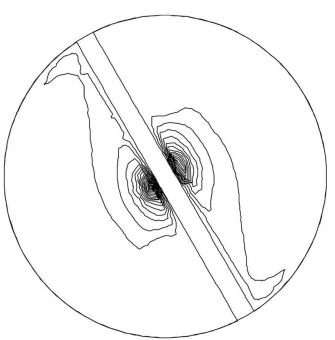

(f) Centrifugal force contours at $30^{\circ}$ twist

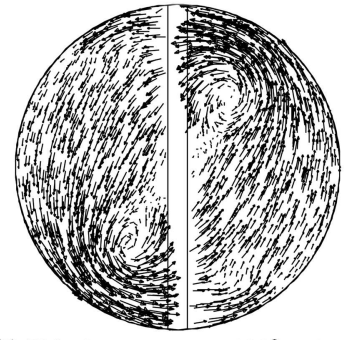

(j) Velocity vectors at $180^{\circ}$ twist (twisted-tape exit plane)

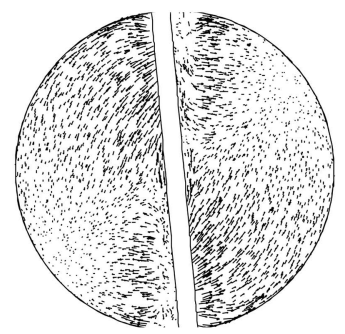

(c) Velocity vectors at $5^{\circ}$ twist ( $2 \mathrm{~mm}$ inside the twisted-tape)

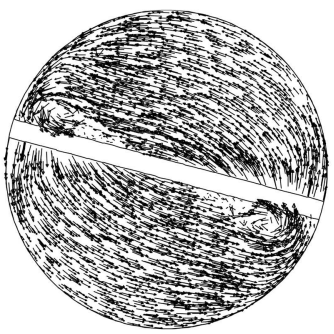

(g) Velocity vectors at $75^{\circ}$ twist (25 $\mathrm{mm}$ inside the twisted-tape)

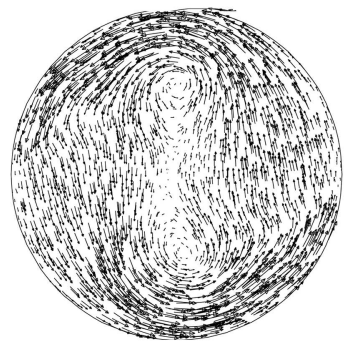

(k) Velocity vectors at $z / d=0.59$ $(15 \mathrm{~mm}$ downstream of the tape exit plane)

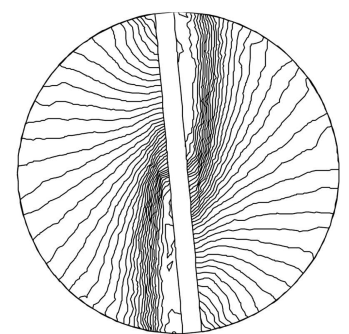

(d) Static pressure contours at $5^{\circ}$ twist

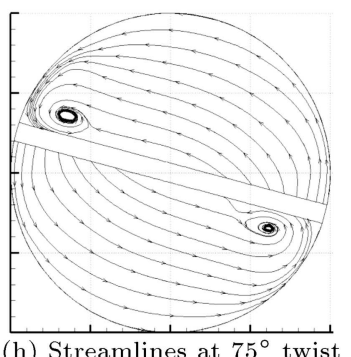

(h) Streamlines at $75^{\circ}$ twist

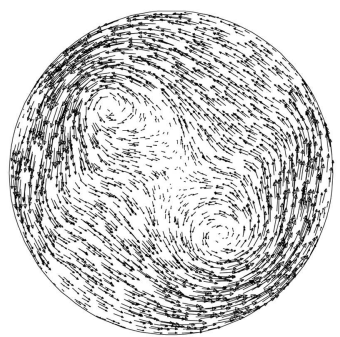

(1) Velocity vectors at $z / d=1.18$ (30 $\mathrm{mm}$ downstream of the tape exit plane)

Figure 17. Vortex formation of a twisted tape with $y_{r}=2.36(60 \mathrm{~mm}$ pitch $)$ at $\operatorname{Re}=7.7 \times 10^{4}$. The cross-section planes inside the twisted tape ( $\mathrm{b}$ to $\mathrm{j}$ ) and downstream of the twisted tape ( $\mathrm{k}$ and $\mathrm{l}$ ) progress from the far end of the twisted tape.

in size. The centrifugal force contours in Figure 17(f) are contours of $\rho V_{\theta}^{2} / r$. After $75^{\circ}$ twist $(25 \mathrm{~mm}$ inside the twisted tape), the centrifugal force on one side and the curved pipe wall on the other side push the streamlines closer together, creating a converging channel and accelerating the flow. The flow acceleration near the pipe wall and the centrifugal ejection move the lowpressure region from the corner of the channel toward the pipe axis, and the flow streamlines are pulled by the low-pressure region until they close into a vortex (Figure 17(g) and (h)). As the vortex co-rotates with the main flow, it also grows in strength as the swirl increases. As it grows, the secondary vortex changes its position inside the semicircular channel in an apparent move against the flow (Figure $17(\mathrm{i})$ and $(\mathrm{j})$ ). This motion against the flow is likely due to conservation of angular momentum as the vortex tends to preserve its rotation axis direction and oppose the change forced by the tape. Downstream of the twisted tape, the two co-rotating vortices maintain their helical formation by rotating with the main flow into the straight pipe
(Figure 17(k) and (1)). The relative position of the vortex at the exit determines the pitch of the helical vortices inside the straight pipe.

\section{Conclusions}

One of the reasons that previous authors proposed a pair of counter-rotating vortex structures inside the twisted tape was based on the fact that measured [3] or simulated [5] axial velocity contours displayed two peaks. Figure 18(a) shows that the axial profile indeed has two peaks; however, these are created by a single co-rotating vortex as shown by the velocity vectors in Figure 18(b).

The smoke visualization of Manglik and Ranganathan [8] at low Re showed one vortex in the direction of rotation; however, the rest of the field did not show the same distinct spiral. The measurements of Seymour were done with a probe inserted in the flow, which could have altered the flow field. Given the perfect match between experiments and the sim- 


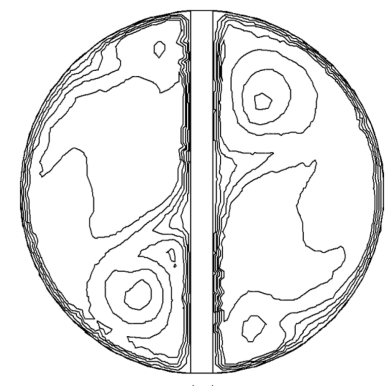

(a)

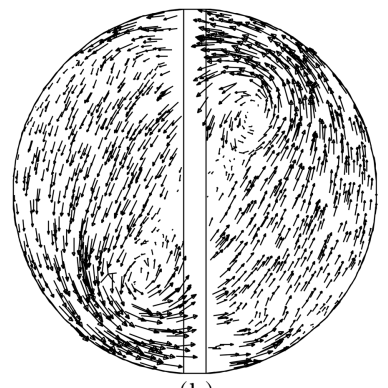

(b)
Figure 18. Axial velocity contours (a) and velocity vectors (b) at, the exit of the twisted tape with $y_{r}=2.36$ at $\operatorname{Re}=7.7 \times 10^{4}$.

ulations presented in this study, the vortex formation mechanism described here appeared to be reliable.

The numerical simulations presented here recovered the characteristics of the helical co-rotating vortices observed in previous experiments [12] and added further details of the flow field. At the same time, the results provided an insight into the formation of the secondary vortices inside the twisted tape, modifying previous hypotheses. The numerical results did not agree with previous studies, reporting the presence of two counter-rotating vortices inside the twisted tape.

A detailed mechanism of the vortex formation was described, showing that the secondary motion was a result of single co-rotating vortices formed on each side of the twisted tape. These vortices result from vortex generation and pressure imbalance created near the inlet of the twisted tape. Moreover, it was shown that the vortex formation was described by DNS better than by the RSM turbulent model.

\section{References}

1. Dewan, A., Mahanta, P., Raju, K.S., and Kumar, P.S. "Review of passive heat transfer augmentation techniques", Proc. Instn. Mech. Engrs., Part A: J. Power and Energy, 218, p. 509 (2004).

2. Kreith, K. and Sonju, O.K. "The decay of a turbulent swirl in a pipe", J. Fluid Mech., 22, p. 257 (1965).

3. Seymour, E.V. "Fluid flow through tubes containing twisted-tapes", The Engineer, 222, p. 634 (1966).

4. Smithberg, E. and Landis, F. "Friction and forced convection heat transfer characteristics in tubes with twisted-tape swirl generators", J. Heat Transfer, 86, p. 39 (1964).

5. Date, A.W. "Prediction of fully-developed flow in a tube containing a twisted-tape", Int. J. Heat Mass Transfer, 17, p. 845 (1974).

6. Manglik, R.M. and Bergles, A.E. "Heat transfer and pressure drop correlations for twisted-tape inserts in isothermal tubes: Part I - laminar flows", J. Heat Transfer, 115, p. 881 (1993).
7. Manglik, R.M., Maramaju, S., and Bergles, A.E. "The scaling and correlation of low Reynolds number swirl flows and friction factors in circular tubes with twistedtape inserts", J. Enhanced Heat Transfer, 8, p. 383 (2001).

8. Manglik, R.M. and Ranganathan, C. "Visualization of swirl flows generated by twisted-tape insert in circular pipes", in Experimental Heat Transfer, Fluid Mechanics and Thermodynamics, M. Giot, F. Mayinger and G.P. Celata Edizioni ETS Pisa, Eds., p. 1631 (1997).

9. Yerra, K.K., Manglik, R.M., and Jog, M.A. "Optimization of heat transfer enhancement in single-phase tubeside flows with twisted-tape inserts", Int. J. Heat Exchangers, 8, p. 117 (2007).

10. Kazuhisa, Y., Hidetoshi, H., and Saburo, T. "Numerical simulation on heat transfer enhancement in twisted-tape-inserted tubes", J. Enhanced Heat Transfer, 11, p. 379 (2004).

11. Patankar, S.V., Numerical Heat Transfer and Fluid Flow, Hemisphere, Washington, DC (1980).

12. Cazan, R. and Aidun, C.K. "Experimental investigation of the swirling flow and the helical vortices induced by a twisted-tape inside a circular pipe", Physics of Fluids, 21, 037102 (2009). https://doi.org/10.1063/1.3085699

13. Alekseenko, S.V., Kuibin, P.A., Okulov, V.L., and Shtork, S.I. "Helical vortices in swirl flow", J. Fluid Mech., 382, p. 195 (1999).

14. Sarpkaya, T. "On stationary and travelling vortex breakdowns", J. Fluid Mech., 45(3), pp. 545-559 (1971).

15. Rahmani, R.K., "Three-dimensional numerical simulation and performance study of an industrial helical static mixer", Ph.D. Thesis, University of Toledo (2004).

16. Rahmani, R.K., Keith, T.G., and Ayasoufi, A. "Threedimensional numerical simulation and performance study of an industrial helical static mixer", J. Fluids Eng., 127, p. 467 (2005).

17. Pope S., Turbulent Flows, Cambridge Press, Cambridge (2000).

18. Holmes, D.G. and Connell, S.D. "Solution of the 2D Navier-Stokes equations on unstructured adaptive grids", AIA A 9th Computational Fluid Dynamics Conference (1989).

19. Rauch, R.D., Batira, J.T., and Yang, N.T.Y. "Spatial adaption procedures on unstructured meshes for accurate unsteady aerodynamic flow computations", Technical Report AIAA-91-1106 (1991).

20. Van Leer, B. "Toward the ultimate conservative difference scheme. A second order sequel to Godunov's method", Journal of Computational Physics, 32, p. 101 (1979). 
21. Issa, R.I. "Solution of the implicitly discretized fluid flow equations by operator-splitting", Journal of Computational Physics, 62, p. 40 (1985).

22. Fluent, FLUENT 6.3 User's Guide, Lebanon, NH (2006). Currently ANSYS Fluent, ANSYS Inc.

23. BLaunder, B.E., Reece, G.J., and Rodi, W. "Progress in the development of a Reynolds-stress turbulence closure", J. Fluid Mech., 68, p. 537 (1975).

24. Launder, B.E. and Spalding, D.B. "The numerical computation of turbulent flows", Computer Methods in Applied Mechanics and Engineering, 3, p. 269 (1974).

25. Robinson, S.K. "Coherent motions in the turbulent boundary layer", Annual Review of Fluid Mechanics, 23, p. 601 (1991).

26. Lugt, H.J. "The dilemma of defining a vortex", in Recent Developments in Theoretical and Experimental Fluid Mechanics, U. Muller, K.G. Roesner, and B. Schmidt, Eds., Springer, Berlin, p. 309 (1979).

27. Jeong, J. and Hussain, A.K.M.F. "On the identification of a vortex", J. Fluid Mech., 285, p. 69 (1995).

28. Aidun, C.K. and Parsheh, M. "Spatially periodic reversing core in a twisted-fin generated swirling pipe flow", Phys. Fluids, 19, 061704 (2007).
29. Islek, A.A. "The impact of swirl in turbulent pipe flow", M.S. Thesis, Georgia Institute of Technology (2004).

\section{Biographies}

Radu Cazan received a BS in Aerospace Engineering from "Politehnica" University in Bucharest and a PhD from the G. W. Woodruff School of Mechanical Engineering at Georgia Institute of Technology in Atlanta. He has worked in the nuclear energy production and oil industries with a focus in fluid mechanics.

Cyrus K. Aidun is a Professor of Fluid Mechanics and Biotransport at the G. W. Woodruff School of Mechanical Engineering and the Parker H. Petit Institute for Bioengineering and Bioscience at Georgia Institute of Technology. He is the Recipient of the National Science Foundation's (NSF) Young Investigator Award, the L.E. Scriven Inaugural Award in liquid coating research, the George Olmsted Award, and the Beloit prize. He has been the program director of the Particulate and Multiphase Processes program at National Science Foundation, where he served in that capacity for a 2-year term. 